\title{
Chapter 1 \\ Fiber Microstructure Sensors Based on Focused Ion Beam Technology
}

\author{
Ricardo Melo André, Manuel J.B. Marques and Orlando Frazão
}

\begin{abstract}
Optical fiber sensors have evolved over the years in many different directions. One particular direction dictated by necessity is miniaturization and the creation of micro- and nano- optical fiber sensors. Many techniques now exist that allow the micro-structuring of optical fiber. One in particular is focused ion beam technology. This chapter aims to introduce this technique and present the latest work on the application of focused ion beam to optical fiber micromachining, more specifically, the fabrication of optical fiber microstructure sensors such as micro-gratings and micro-cavities.
\end{abstract}

\subsection{Introduction}

Optical fiber sensors are fiber devices used to detect different external parameters such as temperature, strain, pressure, acceleration, humidity, chemical concentrations, presence of biological species, and many more [1]. The general principal is that light from a source, such as a laser or a broadband source, is sent down a fiber through the sensing region where it will experience changes in its properties (e.g. wavelength) or propagation conditions (e.g. optical path). This altered light then reaches a detector that measures these changes and correlates them to the respective changes in the external conditions.

Among the most common optical fiber sensors are fiber Bragg gratings (FBGs) [2], which are based on the temperature and mechanical strain dependence of the Bragg wavelength (maximum reflectivity wavelength) of the FBG itself; distributed

\footnotetext{
R.M. André $(\varangle) \cdot$ M.J.B. Marques · O. Frazão

INESC TEC and Faculty of Sciences of the University of Porto (FCUP),

Rua do Campo Alegre, 687, 4150-179 Porto, Portugal

e-mail: rmeloandre@gmail.com

M.J.B. Marques

e-mail: mbmarque@fc.up.pt

O. Frazão

e-mail: ofrazao@fc.up.pt

(C) Springer International Publishing Switzerland 2016

P. Ribeiro and M. Raposo (eds.), Photoptics 2015,

Springer Proceedings in Physics 181, DOI 10.1007/978-3-319-30137-2_1
} 
fiber sensors based on Rayleigh [3], Raman [4], or Brillouin [5, 6] scattering, not dependent on any additional structuring of the fiber; and Fabry-Perot interferometers, which are generally composed of two parallel reflecting surfaces, where interference occurs from light reflected at both interfaces [7]. Many other sensors exist that depend on microstructured optical fibers [8], long period gratings [9], tapers [10], combinations of different fiber types such as SMF-MMF-SMF structures that work as MMI devices [11], and others. What all these devices have in common is that they are mostly based on standard-diameter optical fiber. This is small when compared to conventional electric sensors but sometimes not small enough for specific applications where smaller volumes, shorter lengths, or even difficult access regions are involved. For example, when considering biological applications such as optogenetics [12] or intra-organism and intracellular sensing [13], typical FBGs are too long.

Fabrication of optical fiber sensors with reduced dimensions - micro- and nanosensors and devices - relies on the adoption of novel techniques that were not previously used in optical fiber sensor fabrication or on the use of costumized tested techniques. These can be loosely divided in two categories: low and high resolution techniques. Low resolution techniques allow the rapid decrease in volume of the optical fiber and/or quick access to the light guiding region. These are generally fast but provide a low resolution in the sense that structures with details only down to few tens of microns can be created. They include chemical etching and tapering. Tapering can itself be achieved with the aid of $\mathrm{CO}_{2}$ lasers [14], filament heating [15], electric arc [16], flame brushing [17], or even chemical etching [18, 19]. High resolution techniques allow the creation of fine details in structures in the order of a few microns or even tens or hundreds of nanometers. Since these allow such a high resolution, they are inherently much slower and can only be applied, in a timely fashion, in small sections of fiber. They include femtosecond laser micromachining $[20,21]$ and FIB milling [22-25].

In most cases, the most efficient method of fabricating micro- and nano- fiber devices and sensors, is combining both high and low resolution techniques. First, with the aid of a low resolution technique, reduce the dimensions of the optical fiber down to a few microns or allow easy access to the light guiding region (e.g. core) and then use a high resolution technique to create a truly micro-/nano- structure on the fiber.

This chapter will focus on the high resolution technique called Focused Ion Beam (FIB) and its combination with low resolution techniques such as chemical etching and tapering for the fabrication of optical fiber sensors. Structures such as Fabry-Perot micro-cavities, fiber-top cantilevers, fiber Bragg micro-gratings among others will be explored and detailed.

\subsection{Focused Ion Beam Technology}

Focused Ion Beam (FIB) is a technology that uses a focused ion beam (usually gallium-ion) to image or ablate/mill the substrate on which it is focused or even aid the deposition of another material on the substrate. Focused Ion Beam technology is 
very similar to scanning electron microscopy. The main difference is that the electron beam that scans the sample is replaced by an ion beam. It was developed mainly in the semiconductor industry and for the preparation of transmission electron microscope samples.

\subsubsection{Focused Ion Beam System}

\subsubsection{Sources}

Most ion beam equipment uses liquid-metal ion sources (LMIS), generally gallium-ion sources. The ion beam is generated from the LMIS through a very strong electric field. A tungsten needle is used to heat-up the gallium and take it to its liquid state. The superficial tension and electric field forces lead to the formation of a gallium cone on the needle tip. This cone has a tip with a very small radius of curvature (ca. $5 \mathrm{~nm}$ ) [26]. As the tip gets sharper, the electric field gets stronger until ions are ejected by evaporation. Gallium ion $\left(\mathrm{Ga}^{+}\right)$emission occurs at a typical voltage of $7000 \mathrm{~V}$ [27].

\subsubsection{FIB Column and Chamber}

The produced ions go through a series of apertures, electrostatic lenses and possibly a mass spectrometer in order to select the ions in a specific range of energies and to reduce the ion beam diameter (Fig. 1.1). At the bottom of the column, an electrostatic deflector controls the final trajectory of the beam and the point of impact on the substrate. A supporting system of vacuum pumps is necessary to maintain vacuum inside the column and the working chamber. A typical vacuum of $1 \times 10^{-7}$ mbar is maintained inside the chamber.

\subsubsection{Ion-Substrate Interaction}

When an ion from the beam hits the substrate surface, it loses energy that is transferred to the electrons and atoms of the substrate. Depending on the ion energy, it can interact with the surface in several ways [27, 28]. The following physical processes are just some interactions that can result from bombarding the surface with ions:

- sputtering of the substrate material with atom and ion emission;

- emission of electrons;

- atom dislocation in the substrate;

- phonon emission and consequent heating; 
Fig. 1.1 Diagram of a focused ion beam system

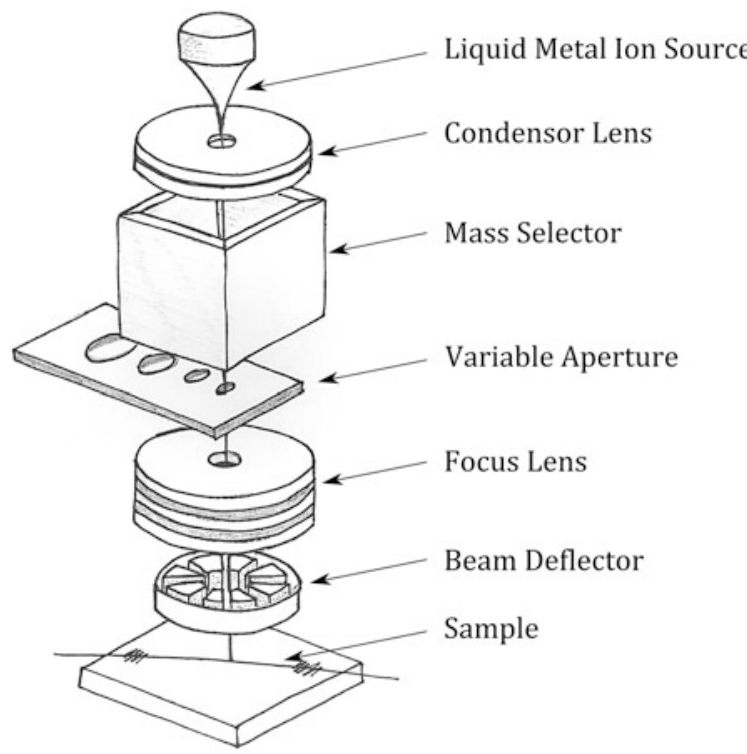

- ion implantation inside the substrate; and

- redeposition of sputtered material.

Focused ion beam can also be used for the deposition of certain materials and in this case, the ion beam is used to break chemical bonds and dissociate gaseous molecules.

\subsubsection{Focused Ion Beam Operation}

Focused Ion Beam systems have three main operation modes: imaging, deposition, and milling.

\section{Imaging}

At low currents, very little material is sputtered and therefore an image of the substrate surface can be obtained without inducing much damage. By scanning the surface, secondary particles will be generated in the substrate; namely atoms, ions and electrons will be ejected from the surface. As they leave the substrate, secondary electrons and sputtered ions are collected by positively and negatively polarized detectors, respectively. The resulting ions can also be guided to a Secondary Ion Mass Spectrometer (SIMS) to obtain the atomic composition of the substrate or even a composition map of the surface of the substrate. Recent FIB systems can easily achieve a $5 \mathrm{~nm}$ resolution limited only by the beam diameter on the substrate and the local sputtering of the surface [26, 27]. 
Obtaining an image with FIB always leads to damage on the surface. During the process, gallium ion implantation and sputtering is inevitable. These two processes can be minimized by reducing beam diameter and current.

\section{Deposition}

Depositing several types of materials from metals to insulators is possible using FIB. The principle behind FIB deposition is Chemical Vapor Deposition (CVD). The main difference is the higher resolution obtained with FIB. This higher resolution comes at the cost of a lower deposition rate. The precursor gases are sprayed to the surface and the molecules are adsorbed onto it. The ion beam will then decompose the precursors and the vacuum system will remove the volatile products that are desorbed from the surface while the desired products of decomposition remain on the surface. The most commonly deposited materials using focused ion beam are tungsten, silver and silicon dioxide [27, 28]. Structures with lateral dimensions of $100 \mathrm{~nm}$ and thickness of $10 \mathrm{~nm}$ can be deposited.

\section{Milling}

Milling designates the process of removing material from the substrate with the aid of the focused ion beam (Fig. 1.2). Physically, it is the combination of two processes: physical sputtering and material re-deposition. It is possible to remove material from the substrate using a high current ion beam. Structures can be created on the substrate by scanning the high current ion beam. The high energy density of the ion beam combined with its small diameter allows the creation of submicron structures. The sputtering yield depends not only on the substrate material but also on the ion energy, incidence angle, and machining conditions. Nonetheless, this yield cannot be considered the etching yield because material re-deposition always occurs reducing the effective etching rate. To accelerate the milling process, a gas may be introduced inside the chamber in order to increase the etching rate. The gas

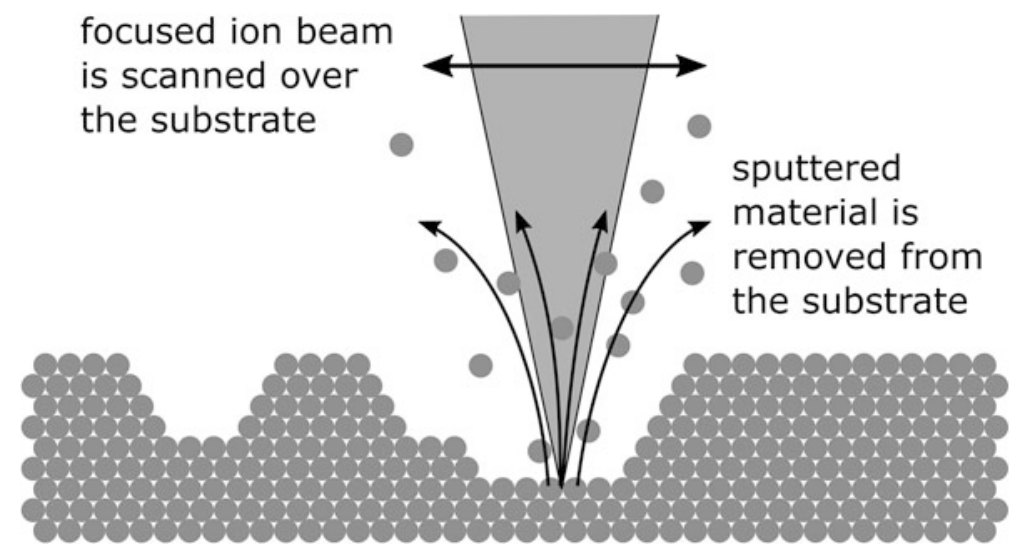

Fig. 1.2 Focused ion beam milling 
will many times increase the etching selectivity and chemically facilitate the removal of reaction products. One of the most developed applications of FIB is the micro-milling of Transmission Electron Microscope (TEM) samples [29].

\subsection{Focused Ion Beam Optical Fiber Applications}

\section{Tapered Fiber Tips}

Of the many structures that can be created using low resolution techniques, the tapered fiber tips are one of the most interesting to use as a base to work with FIB milling. They are important optical devices without any further post-processing but the use of focused ion beam to further develop them can introduce new uses and applications.

A Tapered Fiber Tip (TFT) is a very interesting fiber optic device for sensing. It consists of a microfiber with a taper region that only has one input/output (see Fig. 1.3). Since the input/output of the fiber has a standard diameter of $125 \mu \mathrm{m}$, connecting this device to other optical components is an easy task. The fabrication of this device is relatively simple. Several different techniques can be used such as chemical etching, tapering by $\mathrm{CO}_{2}$ laser heating, tapering by filament heating, etc. When fabricating a taper by heating methods, tension is applied until the fiber is separated into two, not necessarily equal, tips. Too short tips have very large losses due to their non-adiabaticity. This leads to coupling of light to non-confined radiation modes. Too long tips also introduce unwanted problems related to an intrinsic lack of stiffness. Therefore, the length of the tip is a very important factor and must be related to the tip's final application. One other technique used to create tapered fiber tips is denominated dynamic chemical etching. In this technique, a hydrofluoric acid solution is used to chemically etch a cleaved fiber tip into a tapered tip [30]. By controlling the relative position between the acid level and the

Fig. 1.3 Micrographs of tapered fiber tips fabricated by dynamic chemical etching [31]

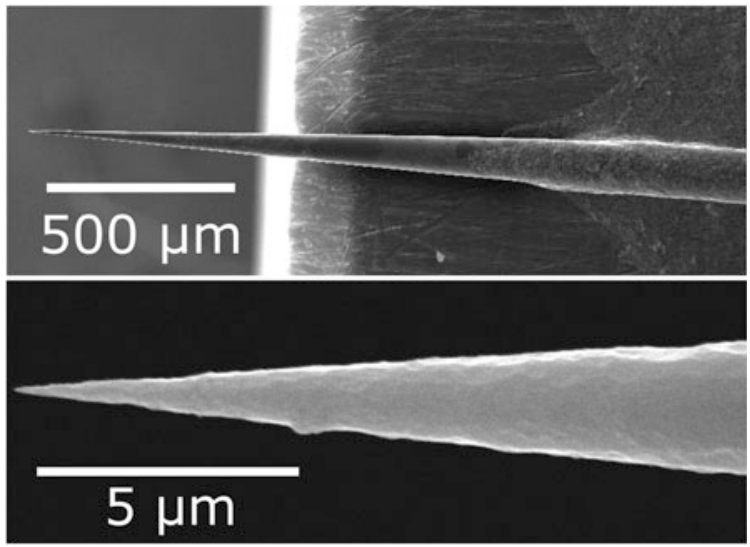


fiber tip, it is possible to control the length, radius, and shape of the TFT. In Fig. 1.3, two examples of tapered fiber tips fabricated by this method are shown.

\subsubsection{Fabry-Perot Micro-Cavity}

One of the simplest structures that one can mill on a tapered fiber tip using FIB is a Fabry-Perot cavity. Using focused ion beam, the cavities are always created removing material in such a way that an open cavity forms. This will result in two surfaces open to the surrounding medium that form the Fabry-Perot cavity.

In literature, several different approaches to cavity creation have been reported. A cavity was created using FIB where a rectangular hole near the cleave of a commercial SMF was milled [22]. As the hole is milled near the cleave, two cavities are created, one in-fiber cavity and one open cavity. This structure is then used for the simultaneous measurement of salinity and temperature. A micro-cavity on a slightly tapered fiber was also explored [32]. The cavity was milled at a diameter of $32 \mu \mathrm{m}$ and used as a refractive index sensor. FIB-milled Fabry-Perot cavities for refractive index sensing were also investigated in polished fibers [24]. A SMF was polished at a very oblique angle to gain access to the core of the fiber, where the cavity was then milled.

More interesting is the milling of microcavities in tapered fiber tips. These can be much smaller than in standard-diameter SMF and due to the point-like design, much more versatile. These structures were applied as temperature [25] and refractive index sensors [33]. In this case, to fabricate the tapered fiber tips, a micropipette puller was used. This basically consists of two micrometric translation stages that stretch the fiber while a $\mathrm{CO}_{2}$ laser is scanned along the fiber to heat it up. For FIB milling, the tip was covered with an aluminum film through evaporation to avoid charge accumulation. In other FIB configurations, a secondary electron beam is used to neutralize the sample and, in this case, the deposition of a conducting film is not necessary. This is an essential step in FIB milling of non-conductive samples (e.g. optical fiber). If the fiber is not in some way prepared to conduct charge out of the milling region, it will inevitably start to charge which will cause the beam to deviate from the desired position. This can cause small deviations in the outcome of the milled structure or even render the milling impossible.

A micro-cavity milled in a TFT is shown in Fig. 1.4. Here, the tip is also cleaved and polished using FIB to create a second silica cavity besides the open cavity. Since the silica cavity is insensitive to external index variations, this structure can be used for the simultaneous measurement of both temperature and refractive index or for temperature-compensated index measurements.

Fabry-Perot cavities have also been milled in exposed core microstructured optical fibers (ECF). The Fabry-Perot cavities were milled directly into the core of ECF [34]. Due to the easy access to the core, it was possible to directly create the cavity on the fiber core without making previous use of any low resolution technique. Two types of cavities where created: one where the milled cavity extends all 

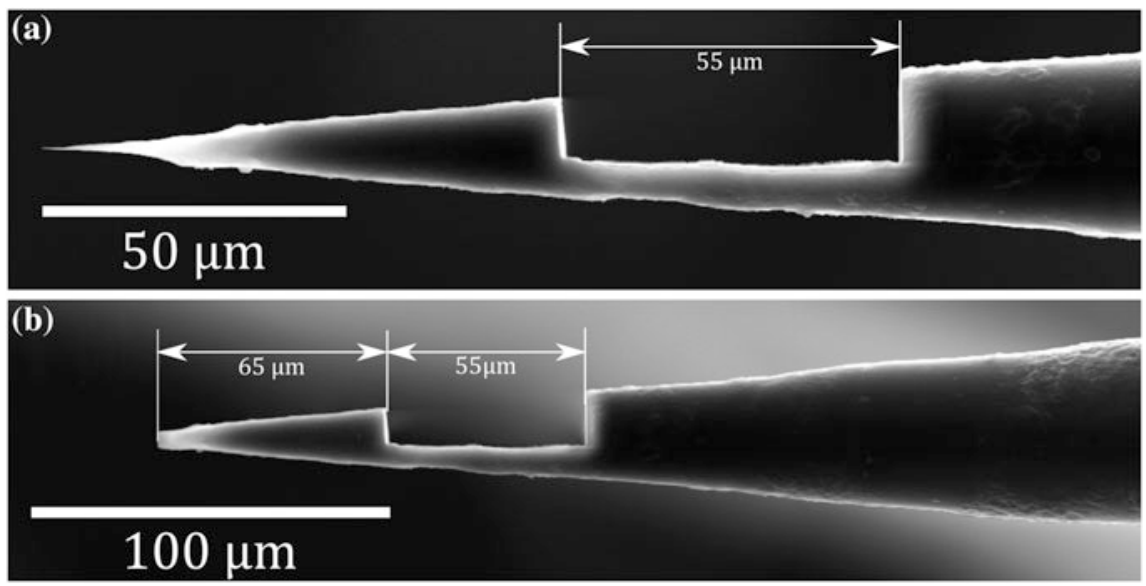

Fig. 1.4 SEM micrograph of a FIB machined micro-cavity on a TFT and $\mathbf{b}$ the same tip with a polished top

the way through the core and allows access to the inner holes of the ECF and a slot cavity that does not reach deep enough and keeps the holes intact. These structures have been proposed for microfluidic sensing. Others had previously applied FIB to photonic crystal fibers but only for accessing specific holes and not sensing [35].

\section{Fiber Cantilevers}

Cantilever structures are devices that rely on the possibility to measure the mechanical deformation of the cantilever itself as a response to a certain external stimulus. The deflection of the cantilever can then be read by electronic or optical methods. Electronic methods may not always be suitable if analyzing conductive liquids, explosive gases and in extreme temperatures for example. Optical methods have a larger range of applicability as long as the medium is optically transparent [36-38].

Fiber top cantilever systems, micro-machined with FIB, have an integrated optical readout - the optical fiber itself. The deflection of the cantilever can be determined by analyzing the signal of the reflected light at the fiber-to-air interface and at the cantilever [36-38]. This is effectively a cavity created between the cantilever and the fiber top. When this cavity is altered, a change in the optical signal is detected. In this case, the readout is done making use of the change in optical power reflected by the fiber-top cantilever. Fiber-top cantilevers are interesting devices that due to their monolithic structure and absence of electronics on the sensing head are ideally suitable for operation in small volumes and also in harsh conditions.

Another cantilever-based system is shown in Fig. 1.5. In this structure, hydrofluoric acid is used to etch special structure forming fibers to create $15 \mu \mathrm{m}$-diameter microwires that are suspended between the input fiber and the fiber top as shown in Fig. 1.5 [39]. Using FIB, the microwire is cleaved and polished close to one end resulting in a suspended microwire-the cantilever. The obtained 

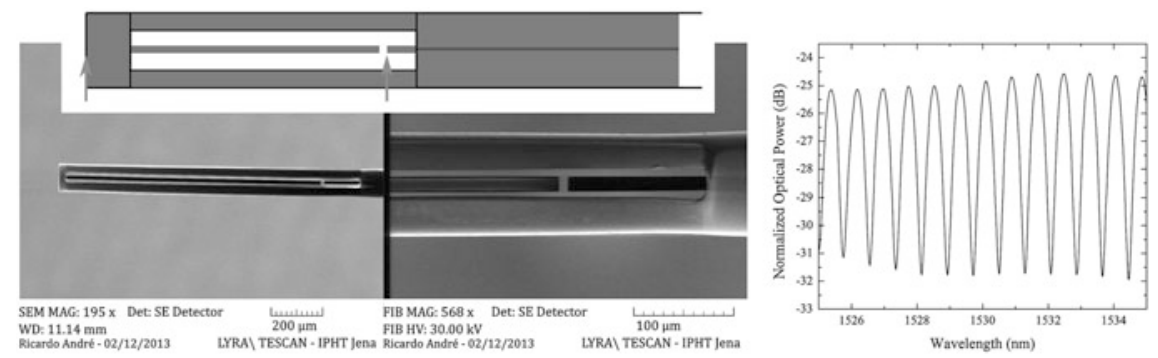

Fig. 1.5 Cantilever Fabry-Perot structure and respective optical spectrum [39]

spectrum is that of a two-wave interferometer. The two surfaces that give rise to this cavity are indicated in Fig. 1.5 by two red arrows.

When the cantilever becomes misaligned with the input fiber, the power reflected by the structure greatly decreases. This inherent property of the structure was used for the creation of a vibration sensor. When a certain vibration frequency is applied to the cantilever structure, the power is modulated accordingly. This can be seen in Fig. 1.6, where the power measured in reflection by a photodiode is represented beside its respective fast Fourier transform for three different applied frequencies.

In the fabrication of this structure, focused ion beam was used to selectively cleave the microwire. This appears to be a simple process but could not be achieved with such a high optical quality with other processing techniques.

\subsubsection{Bragg Micro-grating}

Creating Bragg gratings on standard diameter fibers by the usual core index modulation is certainly the easiest method. But, due to the weak index modulation,
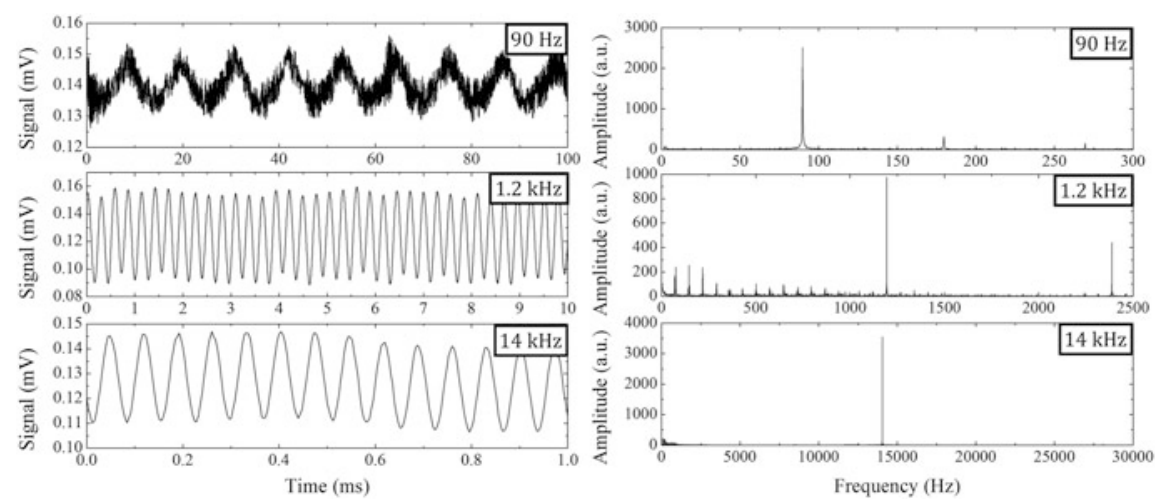

Fig. 1.6 Cantilever Fabry-Perot structure: time responses (left) and respective fast Fourier transforms (right) when an external frequency is applied [39] 
a length of at least several millimeters is necessary. To reduce the length of the grating, a stronger modulation is necessary. This can be done by creating alternate layers of two materials with a high index difference such as silica and air. This would be very difficult to reproduce in standard fibers as it would be necessary to remove large quantities of material just to access the fiber core, where light propagates. In tapers and TFTs such problem does not exist, only small quantities need to be removed to create a grating since light is confined by the silica-air interface. FIB is then the adequate technique for this job. It can easily mill the fiber tips and create a micro-grating. It becomes possible to reduce the grating size from a few millimeters to few tens of micrometers [40].

One example of a micro-grating created with focused ion beam is composed of just $61,600 \mathrm{~nm}$-periods totaling a length of $36.6 \mu \mathrm{m}$ [40]. This grating has a corrugation depth of $200 \mathrm{~nm}$ and was milled in a region with an approximate radius of $3.25 \mu \mathrm{m}$. This micro-grating was characterized as a temperature sensor and was subjected to temperature variations from room-temperature $\left(21^{\circ} \mathrm{C}\right)$ to $440{ }^{\circ} \mathrm{C}$ and a mean sensitivity of approximately $20 \mathrm{pm} / \mathrm{K}$ was obtained [40]. An example of even smaller dimensions a micro-grating with a length of just $12 \mu \mathrm{m}$, composed of just 11 indentations created on a TFT [41].

A Bragg micro-grating on a TFT is shown in Fig. 1.7. This grating has a total of 50 periods with corrugations $270 \mathrm{~nm}$-long and $1.3 \mu \mathrm{m}$-deep, totaling a length of $27 \mu \mathrm{m}$ [31]. The grating is created in a section with a diameter range of [2.7, 5.1] $\mu \mathrm{m}$. Using a current of just $115 \mathrm{pA}$, this grating takes ca. $50 \mathrm{~min}$ to structure (TESCAN LYRA FIB-SEM dual-beam system).

This grating is highly sensitive to the external medium where it is placed because the index modulation changes completely from, for example, air-silica modulation (1-1.44) to isopropanol-silica modulation (1.3-1.44) (see Fig. 1.8).

One of the great advantages of this Bragg grating configuration is its small length. Since it is much smaller than usual, it is much easier to manipulate without inducing curvature or stress that would lead to undesired effects when measuring temperature. Furthermore, as opposed to gratings produced by UV exposure where the index modulation is weak and degrades at temperatures higher than $200{ }^{\circ} \mathrm{C}$ due to germanium diffusion, these FIB milled gratings can withstand much higher temperatures as the modulation is geometric in nature and only temperatures close to the fusion of silica can lead to its degradation.

Fig. 1.7 SEM micrograph of a Bragg grating created on a TFT [31]

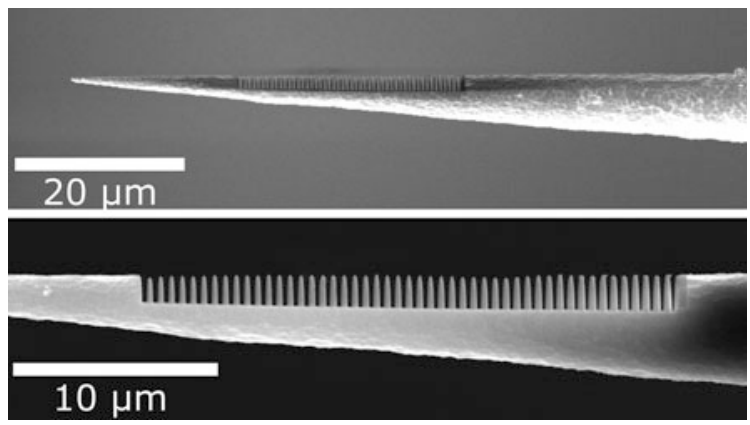


Fig. 1.8 Bragg micro-grating spectra when dipped in air (top) and in isopropanol (bottom) [31]

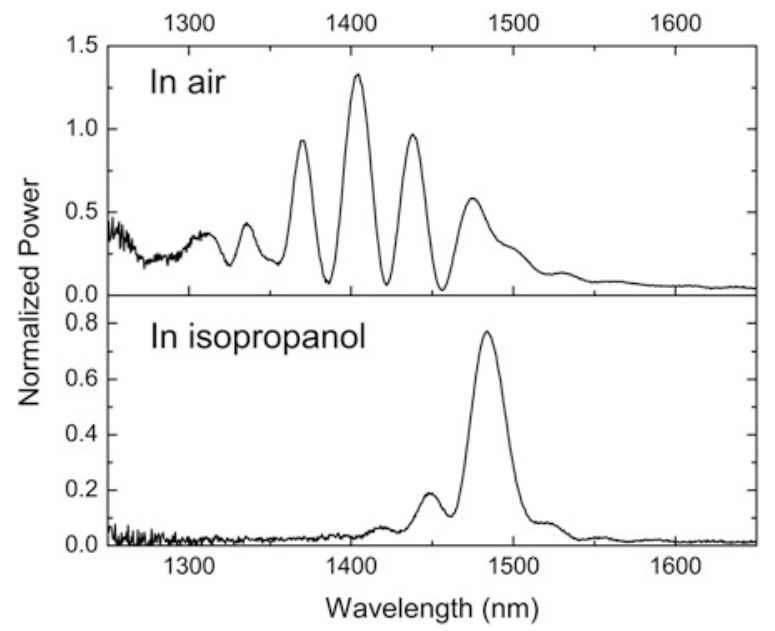

\subsection{Concluding Remarks}

It was shown that focused ion beam technology has a large potential when it comes to the fabrication of optical fiber sensor microstructures. The tendency is to use focused ion beam in the fabrication of an increasing number of new micro- and nanostructures. The push towards smaller devices into the nanometer range is still active but other areas will start being explored. Presently one can already see focused ion beam being combined with microstructured optical fibers, tapers, and chemically etched structures for the sensing of physical parameters such as temperature and refractive index but soon many others will follow due to the huge potential of this technology in fiber sensing. In the near future, combining focused ion beam post-processing and the functionalization of the milled structures and surfaces will be essential for biosensing. This combination has a large potential as it takes the advantages of miniaturization: smaller devices, faster responses, higher sensitivities and adds biospecificity to create novel micro- and nano-sensors in the ever-expanding field of biosensing.

\section{References}

1. Santos, J.L., Farahi, F.: Handbook of optical sensors. CRC Press, USA (2014)

2. Kersey, A.D., Davis, M.A., Patrick, H.J., LeBlanc, M., Koo, K.P., Askins, C.G., Putnam, M. a., Friebele, E.J.: Fiber grating sensors. J. Light. Technol. 15, 1442-1463 (1997)

3. Barnoski, M.K., Rourke, M.D., Jensen, S.M., Melville, R.T.: Optical time domain reflectometer. Appl. Opt. 16, 2375 (1977)

4. Dakin, J.P., Pratt, D.J., Bibby, G.W., Ross, J.N.: Distributed optical fibre Raman tempera-ture sensor using a semiconductor light source and detector. Electron. Lett. 21, 569 (1985) 
5. Horiguchi, T., Kurashima, T., Tateda, M.: Tensile strain dependence of Brillouin frequency shift in silica optical fibers. IEEE Photonics Technol. Lett. 1, 107-108 (1989)

6. Kurashima, T., Horiguchi, T., Tateda, M.: Distributed-temperature sensing using stimulated Brillouin scattering in optical silica fibers. Opt. Lett. 15, 1038 (1990)

7. Kersey, A.D., Jackson, D.A., Corke, M.: A simple fibre Fabry-Perot sensor. Opt. Commun. 45, 71-74 (1983)

8. André, R.M., Marques, M.B., Roy, P., Frazao, O.: Fiber loop mirror using a small core microstructured fiber for strain and temperature discrimination. IEEE Photonics Technol. Lett. 22, 1120-1122 (2010)

9. Bhatia, V., Vengsarkar, A.M.: Optical fiber long-period grating sensors. Opt. Lett. 21, 692 (1996)

10. Birks, T.A., Li, Y.W.: The shape of fiber tapers. J. Light. Technol. 10, 432-438 (1992)

11. André, R.M., Biazoli, C.R., Silva, S.O., Marques, M.B., Cordeiro, C.M.B., Frazao, O.: Strain-temperature discrimination using multimode interference in tapered fiber. IEEE Photonics Technol. Lett. 25, 155-158 (2013)

12. Deisseroth, K.: Optogenetics. Nat. Methods 8, 26-29 (2011)

13. Tan, W., Shi, Z.Y., Smith, S., Birnbaum, D., Kopelman, R.: Submicrometer intracellular chemical optical fiber sensors. Science 258, 778-781 (1992)

14. Ozcan, L.E., Treanton, V., Guay, F., Kashyap, R.: Highly symmetric optical fiber tapers fabricated with a $\mathrm{CO}_{2}$ laser. IEEE Photonics Technol. Lett. 19, 656-658 (2007)

15. André, R.M.: High birefringence triangular optical nanowire in suspended-core fiber for temperature sensing. J. Nanophotonics 7, 073088 (2013)

16. Rego, G.: Fibre optic devices produced by arc discharges. J. Opt. 12, 113002 (2010)

17. Kenny, R.P., Birks, T.A., Oakley, K.P.: Control of optical fibre taper shape. Electron. Lett. 27, 1654 (1991)

18. Kbashi, H.J.: Fabrication of Submicron-diameter and taper fibers using chemical etching. J. Mater. Sci. Technol. 28, 308-312 (2012)

19. Dong, L., Cruz, J.L., Reekie, L., Tucknott, J.A.: Chirped fiber bragg gratings fabricated using etched tapers. Opt. Fiber Technol. 1, 363-368 (1995)

20. Wei, T., Han, Y., Li, Y., Tsai, H.-L., Xiao, H.: Temperature-insensitive miniaturized fiber inline Fabry-Perot interferometer for highly sensitive refractive index measurement. Opt. Express 16, 5764 (2008)

21. Liao, C.R., Hu, T.Y., Wang, D.N.: Optical fiber Fabry-Perot interferometer cavity fabricated by femtosecond laser micromachining and fusion splicing for refractive index sensing. Opt. Express 20, 22813-22818 (2012)

22. Nguyen, L.V., Vasiliev, M., Alameh, K.: Three-wave fiber fabry-pérot interferometer for simultaneous measurement of temperature and water salinity of seawater. IEEE Photonics Technol. Lett. 23, 450-452 (2011)

23. Yuan, L., Yang, J., Liu, Z., Sun, J.: In-fiber integrated Michelson interferometer. Opt. Lett. 31, 2692 (2006)

24. Wieduwilt, T., Dellith, J., Talkenberg, F., Bartelt, H., Schmidt, M.A.: Reflectivity enhanced refractive index sensor based on a fiber-integrated Fabry-Perot microresonator. Opt. Express 22, 25333-25346 (2014)

25. Kou, J., Feng, J., Ye, L., Xu, F., Lu, Y.: Miniaturized fiber taper reflective interferometer for high temperature measurement. Opt. Express 18, 14245-14250 (2010)

26. Volkert, C.A., Minor, A.M.: Focused ion beam micromachining. Mater. Res. Soc. Bull. 32, 389-399 (2007)

27. Reyntjens, S., Puers, R.: A review of focused ion beam applications in microsystem technology. J. Micromech. Microeng. 11, 287-300 (2001)

28. Tseng, A.A.: Recent developments in micromilling using focused ion beam technology. J. Micromech. Microeng. 14, R15-R34 (2004)

29. Giannuzzi, L.A., Stevie, F.A.: A review of focused ion beam milling techniques for TEM specimen preparation. Micron 30, 197-204 (1999) 
30. Nikbakht, H., Latifi, H., Amini, T., Chenari, Z.: Controlling cone angle of the tapered tip fiber using dynamic etching. In: OFS2014 23rd International Conference on Optical Fiber Sensors (2014)

31. André, R.M., Becker, M., Dellith, J., Rothhardt, M., Zibaii, M.I., Latifi, H., Marques, M.B., Bartelt, H., Frazão, O.: Bragg grating fabrication on tapered fiber tips based on focused ion beam milling. In: OFS2015 24th International Conf. on Optical Fiber Sensors (2015)

32. Yuan, W., Wang, F., Savenko, A., Petersen, D.H., Bang, O.: Note: Optical fiber milled by focused ion beam and its application for Fabry-Pérot refractive index sensor. Rev. Sci. Instrum. 82, 076103 (2011)

33. Kou, J., Feng, J., Wang, Q., Xu, F., Lu, Y.: Microfiber-probe-based ultrasmall interferometric sensor. Opt. Lett. 35, 2308 (2010)

34. Warren-Smith, S.C., André, R.M., Perrella, C., Dellith, J., Rothhardt, M., Bartelt, H.: Direct core structuring of microstructured optical fibers using focused ion beam milling. Opt. Express. 24, 378-387 (2016)

35. Wang, F., Yuan, W., Hansen, O., Bang, O.: Selective filling of photonic crystal fibers using focused ion beam milled microchannels. Opt. Express 19, 17585 (2011)

36. Iannuzzi, D., Deladi, S., Schreuders, H., Slaman, M., Rector, J.H., Elwenspoek, M.: Fiber-top cantilevers: a new generation of micromachined sensors for multipurpose applications-OSA technical digest (CD). In: Optical Fiber Sensors. p. TuB2. Optical Society of America, USA (2006)

37. Iannuzzi, D., Deladi, S., Gadgil, V.J., Sanders, R.G.P., Schreuders, H., Elwenspoek, M.C.: Monolithic fiber-top sensor for critical environments and standard applications. Appl. Phys. Lett. 88, 053501 (2006)

38. Iannuzzi, D., Heeck, K., Slaman, M., de Man, S., Rector, J.H., Schreuders, H., Berenschot, J. W., Gadgil, V.J., Sanders, R.G.P., Elwenspoek, M.C., Deladi, S.: Fibre-top cantilevers: design, fabrication and applications. Meas. Sci. Technol. 18, 3247-3252 (2007)

39. André, R.M., Pevec, S., Becker, M., Dellith, J., Rothhardt, M., Marques, M.B., Donlagic, D., Bartelt, H., Frazão, O.: Focused ion beam post-processing of optical fiber Fabry-Perot cavities for sensing applications. Opt. Express 22, 13102-13108 (2014)

40. Kou, J., Qiu, S., Xu, F., Lu, Y.: Demonstration of a compact temperature sensor based on first-order Bragg grating in a tapered fiber probe. Opt. Express 19, 18452 (2011)

41. Feng, Jing, Ding, Ming, Kou, Jun-Long, Fei, Xu, Yan-Qing, Lu: An optical fiber tip micrograting thermometer. IEEE Photon. J. 3, 810-814 (2011) 